\title{
Visions of Nature's Planet Foundry: Images of Circumstellar Disks
}

\author{
Karl Stapelfeldt \\ MS 183-900, Jet Propulsion Laboratory, California Institute of \\ Technology, Pasadena CA 91109, USA; krs@exoplanet.jpl.nasa.gov
}

\begin{abstract}
As the number of detected extrasolar planetary systems has steadily grown over the past five years, so too has the number of circumstellar disks with resolved images. In this contribution, I take stock of the current inventory of disk images at various wavelengths; summarize the results of a new disk imaging survey conducted with the Hubble Space Telescope; review the major inferences that can be drawn about disk structure from the extant images; and suggest areas for future progress.
\end{abstract}

\section{Introduction and Background}

Circumstellar disks are the builders of worlds; they spin ordinary interstellar gas and dust into astronomical gold, the planetary systems which play host to life in the universe. In the first 10 million years of a star's life its dense natal disk dissipates, leaving behind a tenuous debris disk and, at least in some cases, a planetary system. In order to understand this key chapter in the story of our origins, astronomers seek detailed views of circumstellar disks - nature's planet foundries. Remarkable advances in disk imaging science have taken place in the past decade, and the field is poised for further progress.

The first well-resolved image of a circumstellar disk was made in scattered light using optical coronagraphy in 1984 ( $\beta$ Pictoris; ref 41 ). Although it was expected that many more disk images would soon follow, optical imaging surveys did not succeed in detecting disks among other main sequence stars with infrared excess (42). Instead, it was millimeter interferometers which obtained the next new disk images, but for much denser young stellar object disks such as that of HL Tauri (17). Subsequent kinematic studies of millimeter molecular line emission were instrumental in showing that disk gas moves in Keplerian orbits about the central star $(9,16,30)$. The advent of the Hubble Space Telescope (HST) rejuvenated optical disk imaging $(3,4,18)$, and together with adaptive optics (AO) on large ground-based telescopes, has provided images of several young disks in the near-infrared $(35,39 ; 37,33)$. Finally, high-resolution thermal infrared imaging has begun to make important contributions to the field $(20,23)$.

Circumstellar disks are usually broken down into two major categories: dense, optically thick young disks associated with pre-main sequence objects such as T Tauri and Herbig Ae stars; and more tenuous, optically thin debris disks associated with nearby, much older main sequence stars. The young disks are gas rich, while the debris disks are dust-dominated; and correspondingly, the young disks have much larger masses comparable to the theoretical mass $(0.01$ 


\begin{tabular}{|c|c|c|c|c|c|}
\hline $\begin{array}{l}\text { Object } \\
\text { Name }\end{array}$ & $\begin{array}{l}\text { Scattered } \\
\text { Light }\end{array}$ & $\begin{array}{l}\text { Thermal } \\
\text { Infrared }\end{array}$ & $\begin{array}{l}\text { Millimeter } \\
\text { Continuum }\end{array}$ & $\begin{array}{l}\text { Millimeter } \\
\text { Lines }\end{array}$ & $\begin{array}{c}\text { Recent } \\
\text { References }\end{array}$ \\
\hline$\epsilon$ Eridani & no & yes & YES & no & $21,2,14$ \\
\hline Fomalhaut & no & yes & YES & no & $21,2,8$ \\
\hline Vega & no & yes & yes & no & $21,2,19$ \\
\hline$\beta$ Pictoris & YES & YES & yes & no & $22,18,34,19$ \\
\hline HR 4796 & YES & YES & no & no & $39,20,23$ \\
\hline HD 141569 & YES & yes & no & no & 39 \\
\hline TW Hydrae & YES & no & yes & no & $25,39,48$ \\
\hline HD 100546 & YES & yes & no & no & 1 \\
\hline HD 163296 & YES & no & no & yes & 13,29 \\
\hline AB Aurigae & yes & yes & no & yes & 31,29 \\
\hline AS 209 & no & no & no & yes & 24 \\
\hline BP Tauri & no & no & no & yes & 41 \\
\hline CI Tauri & no & no & yes & yes & 10 \\
\hline CY Tauri & no & no & yes & yes & 10,41 \\
\hline DG Tauri B & yes & no & yes & yes & 35,44 \\
\hline DL Tauri & no & no & yes & yes & 10,41 \\
\hline DM Tauri & no & no & no & YES & 16 \\
\hline DN Tauri & no & no & yes & no & 10 \\
\hline DO Tauri & no & no & no & yes & 24 \\
\hline FT Tauri & no & no & yes & yes & 10 \\
\hline GG Tauri & YES & no & YES & YES & $37,40,15$ \\
\hline GM Aurigae & YES & no & yes & YES & 44,9 \\
\hline GO Tauri & yes & no & no & yes & 44 \\
\hline HH 30 & YES & no & yes & yes & $4,44,45$ \\
\hline HK Tauri B & YES & no & yes & no & 46 \\
\hline HL Tauri & yes & no & yes & YES & $7,26,17$ \\
\hline HV Tauri C & YES & no & no & no & 33 \\
\hline Haro 6-5B & YES & no & no & yes & 35 \\
\hline IRAS $04302+2247$ & YES & no & YES & YES & 35 \\
\hline L1551 IRS 5 & no & no & yes & no & 38 \\
\hline $\mathrm{LkCa} 15$ & no & no & no & YES & 11 \\
\hline MWC 480 & no & no & no & YES & 30,41 \\
\hline MWC 758 & no & no & no & yes & 28 \\
\hline RY Tauri & no & no & no & yes & 24 \\
\hline UY Aurigae & YES & no & no & yes & 6,12 \\
\hline UZ Tauri e & no & no & yes & yes & 41 \\
\hline Schwartz 82 & YES & no & no & no & 44 \\
\hline Orion 114-426 & YES & no & no & no & 3 \\
\hline Orion $121-1925$ & yes & no & no & no & 3 \\
\hline Orion $132-1832$ & yes & no & no & no & 3 \\
\hline Orion $163-026$ & yes & no & no & no & 3 \\
\hline Orion $165-254$ & yes & no & no & no & 3 \\
\hline Orion $167-231$ & yes & no & no & no & 3 \\
\hline Orion $172-028$ & yes & no & no & no & 3 \\
\hline Orion $182-332$ & yes & no & no & no & 3 \\
\hline Orion $182-413$ & yes & no & no & no & 3 \\
\hline Orion $183-405$ & YES & no & no & no & 3 \\
\hline
\end{tabular}




\begin{tabular}{lccccc}
\hline $\begin{array}{l}\text { Object } \\
\text { Name }\end{array}$ & $\begin{array}{c}\text { Scattered } \\
\text { Light }\end{array}$ & $\begin{array}{c}\text { Thermal } \\
\text { Infrared }\end{array}$ & $\begin{array}{c}\text { Millimeter } \\
\text { Continuum }\end{array}$ & $\begin{array}{c}\text { Millimeter } \\
\text { Lines }\end{array}$ & $\begin{array}{c}\text { Recent } \\
\text { References }\end{array}$ \\
\hline Orion 191-232 & yes & no & no & no & 3 \\
Orion 203-506 & yes & no & no & no & 3 \\
Orion 218-354 & YES & no & no & no & 3 \\
Orion 218-529 & yes & no & no & no & 3 \\
Orion 239-334 & yes & no & no & no & 3 \\
Orion 294-606 & yes & no & no & no & 3 \\
\hline \hline
\end{tabular}

Table 1. An inventory of resolved circumstellar disks.

A 'YES' entry indicates that the disk is well-resolved at this wavelength; a 'yes' entry means the disk is marginally resolved, with only its outer radius, position angle, and inclination measured. Debris disks are listed in the top section, and young stellar object disks in the lower section. Sources are listed in order of heliocentric distance. An updated version of this table will be kept at http://exoplanet.jpl.nasa.gov/diskimcat.

$\mathrm{M}_{\odot}$ ) for the solar nebula which spawned our own Sun's planetary system. Small dust grains, similar to their ISM progenitors, are plentiful in the young disks; whereas in debris disks, larger second-generation grains derived from planetesimal collisions appear to dominate (2). As planet formation likely takes place during the evolutionary transition between the two kinds of disks, there is great interest in indentifying intermediate cases.

Table 1 lists the spatially resolved circumstellar disks known to the author as of late 2000. Not listed are unpublished results, and objects for which the disk detection is in doubt (such as 55 Cancri; refs. 47 and 39). Disks are suspected for many more objects than listed in this table (such as main sequence stars with infrared excess, and externally ionized Orion Nebula "proplyds"); however, only those with resolved images are considered here. The current inventory of imaged disks is at least 53 objects. Twenty-four are well-resolved, with significant internal structure visible. There are 47 resolved disks associated with young stellar objects, and only 6 resolved debris disks. Optical and nearIR imaging has produced the largest number of disk images, followed closely by $\mathrm{mm} / \mathrm{submm}$ techniques. Only two disks are well-resolved in three different observational regimes. A representative set of disk images is shown in Fig. 1.

\section{An HST Survey of Nearby T Tauri Disks}

Disk images at high spatial resolution and high image contrast can be made in optical and near-IR reflected light using the HST. The first deep imaging survey of T Tauri stars with HST, conducted with the WFPC2 camera in two snapshot observing programs, has just completed (44). The sample consisted of 134 young stars associated with the Taurus-Auriga, Chamaeleon, Lupus, Ophiuchus, and Corona Australis star-forming clouds at distances ranging from 140-160 pc. Most of the targets possess millimeter continuum emission indicating the presence of circumstellar disks, and/or optical polarization suggesting the presence of compact reflection nebulosity.

A wide variety of circumstellar nebulae have been found in this data set. More than a dozen sources show extended nebulosity reminiscent of circumstellar envelopes, with appearances ranging from symmetric bipolar cavities to 


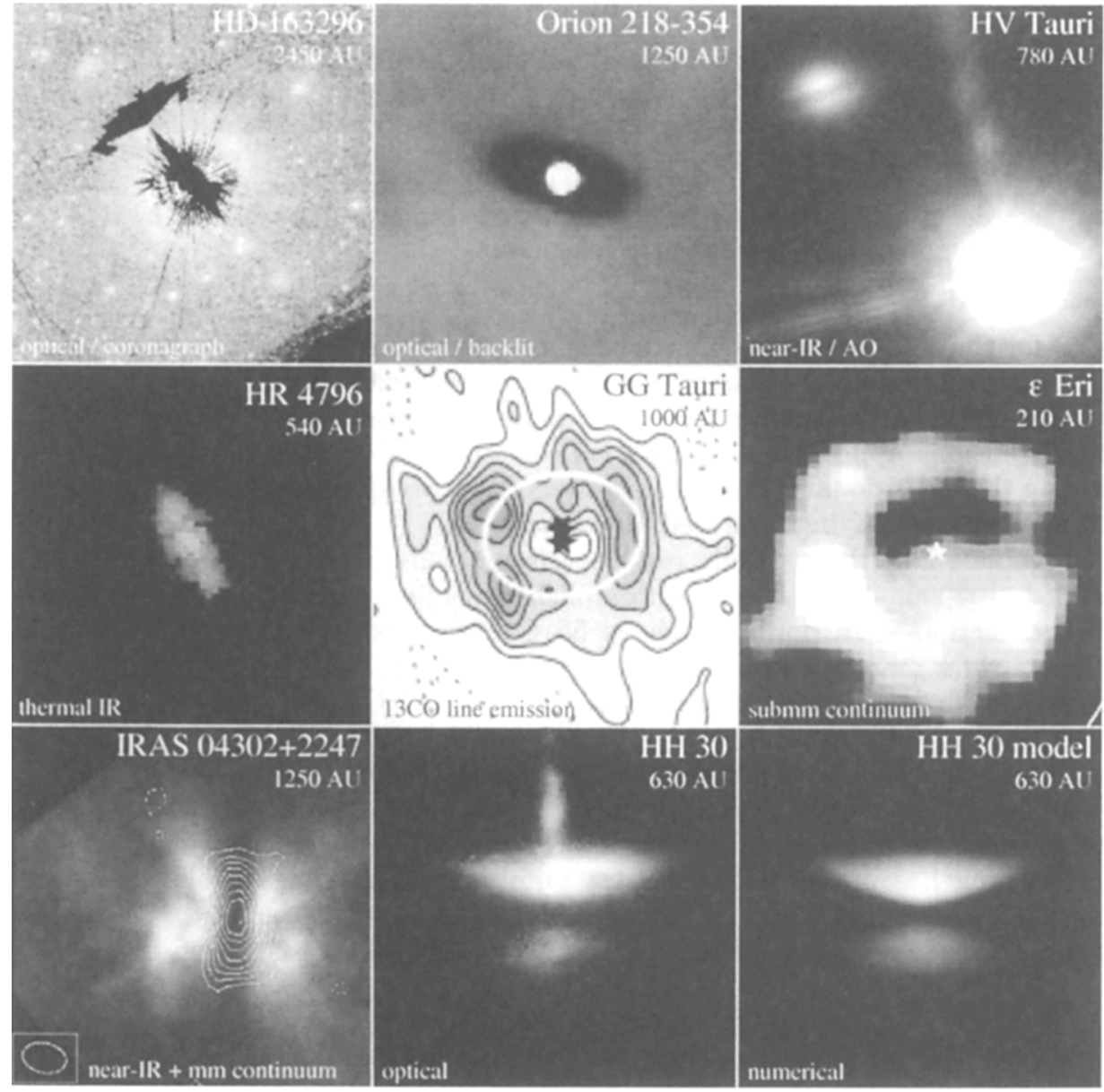

Figure 1. Images of circumstellar disks. Each panel is labeled with the object name, wavelength, and the size of the image field of view. From left to right in each row, the image sources are references top: (13), (3), (33); center: (23), (15), (14); and bottom: (35), (4), (4). 
irregular structures without obvious interpretation. Jet emission ranging from diffuse patches to compact knots is seen in six objects. Most interesting are eight sources where HST directly detects the disks in scattered light. These include the edge-on disks in the HK Tau (46) and HV Tau binary systems; the circumbinary ring in UY Aur; intermediate inclination disks associated with GM Aur, GO Tau, Sz 82, and DoAr 25; and a face-on disk around TW Hya (25). Modeling of the HK Tau and TW Hya images has found a rough order-ofmagnitude agreement in disk mass estimates independently derived at optical and millimeter wavelengths. A similar agreement was also found in the case of $\mathrm{HH} 30$ (4). This suggests that there has been no major depletion of micron-sized dust grains in the outer regions of these disks, and thus any planet formation process must still be in its earliest stage.

For two-thirds of the targets observed, however, HST/WFPC2 detects no nebulosity at all: only the stellar point spread function is seen. This includes 16 objects where millimeter interferometers have clearly resolved disks larger than 3 arcsec in diameter, and an additional 32 sources with millimeter continuum emission. Adaptive optics surveys have also failed to detect the scattered light from a similar but smaller sample of disks surveyed to date. A number of possibilities are available to explain why these disks are undetected in scattered light, including unfavorable orientation, a highly flattened geometry, and evolution in dust particle properties.

Finally, the number of edge-on disks detected in the survey was lower than expected. Geometrical arguments suggest that $\sim 15 \%$ of randomly oriented, optically thick disks should occult their central star, yet only 2/134 were found in this survey. Since an edge-on disk diminishes the total flux from the system by 3-5 mags, it is clear that existing $T$ Tauri star catalogs suffer from a selection bias against these objects. It is likely that a dozen young edge-on disks remain to be identified in nearby molecular cloud complexes.

\section{Trends in Disk Structure}

Disk geometry, density, temperature distribution, and dust properties are best constrained through detailed comparisons of data with model images. Notable recent examples of this approach include multiwavelength image modeling of the GG Tau circumbinary ring $(15,49) ; \chi^{2}$-optimized model fitting to images of HH 30's edge-on disk (4); and combined image and spectral energy distribution modeling of the HR 4796 and Fomalhaut debris disks $(23,8)$. Key results of these and other efforts can be broken down in the following way:

Disk Radial Structure. All of the resolved disks have a radial extent comparable to or larger than our solar system's Kuiper Belt. In several cases, outer radii extending beyond $500 \mathrm{AU}$ have been found $(16,22,35)$. Central holes cleared by tidal interactions have been directly imaged in young circumbinary disks $(37,15,6)$. They have also been imaged in debris disks; however, it is not known if the central dust clearing in these cases has been accomplished by dynamical or radiative processes. Several debris disks have noteworthy radial structure: HR 4796, $\epsilon$ Eri, and Fomalhaut have been resolved as relatively narrow rings $(39,14,8)$, while HD 141569 has a prominent radial gap (39). Radial density gradients have proven difficult to infer in optically thick young disks. 
Submillimeter results for HL Tau favor centrally concentrated surface densities declining with radius (26). The disk with the best-determined radial density gradient is that of $\beta$ Pic, where a $\mathrm{r}^{-3}$ surface density law is indicated by the scattered light brightness profile $(22,18)$.

Disk Vertical Structure. Vertical profiles can be studied in edge-on disk systems. Flaring (i.e., increasing disk thickness with radius) is now directly observed in young stellar object disks. Detailed studies of the HH 30 disk indicate a Gaussian vertical profile with a scale height comparable to that expected from local thermal equilibrium $(4,50)$. However, a smaller than expected scale height has been measured in the disk of HK Tau B (46), possibly indicating that the dust has settled with respect to the gas in this disk. In debris disks, where gas is largely absent, the disk vertical profile traces the orbital inclination distribution for the parent bodies whose collisions are replenishing the dust population. An unusually thick torus is indicated in the case of Fomalhaut (8). Conversely, the disk of $\beta$ Pic is especially thin, with an approximately exponential vertical profile (5), and a warped inner region which may indicate the presence of a perturbing body near its inner edge (34).

Disk Asymmetries. Azimuthal asymmetries cannot persist in a disk for more than a few orbital timescales before being disrupted by Keplerian shear. Therefore, the presence of such asymmetries strongly suggests that a disk is being perturbed by some other object. The $\beta$ Pic disk has several subtle asymmetries such as differences in its radial brightness profile and vertical thickness between the NE and SW extensions (22); one possible explanation is that the disk was recently disturbed by the close passage of an as-yet-unidentified $\mathrm{M}$ dwarf star. The $\epsilon$ Eri dust ring appears to have major azimuthal clumps and voids which can plausibly be forced by a jovian planet orbiting near or within the ring (27). Finally, a most unusual variable brightness asymmetry has been observed in optical images of HH 30: a bright spot of light appears to be moving around the disk (45). While this may be produced by stellar hotspot illumination, another viable model is beaming/shadowing of the outer disk as starlight propagates through azimuthal inhomogeneities in the optically thick inner disk.

\section{Future Prospects}

There is much room for growth in the field of disk imaging science in the coming decade. Specific activities that would advance the field include: 1) Expand the inventory of disks imaged at all wavelengths and in all stellar contexts. New debris disk images are especially needed, as only six of these objects have been resolved to date. 2) Make greater efforts to derive disk structure parameters by comparing models to multiwavelength resolved images and spectral energy distributions. Detailed modeling work has not kept pace with the growing number of well-resolved disk images. 3) Map the radial gradients in gas chemistry and dust properties within disks, to document the evolution from interstellar to planetary material. Imaging evidence of dust settling to a disk's midplane should be obtainable in young edge-on disks. 4) Establish the presence and distribution of dust in our own Kuiper Belt, for direct comparison to external debris disk systems. Perhaps the ultimate goal is 5) To locate planets within resolved disks and relate them to disk radial structures and asymmetries. 
To accomplish these goals, improvements will be needed in observational methods at all wavelengths. In scattered light and thermal infrared images, the most tenuous disk reliably imaged to date is still that of $\beta$ Pic - with $10^{4}$ times the vertical optical depth of our solar system's zodiacal light. In the optical and near-infrared, detection of more tenuous disks will require much better techniques for high contrast imaging. In the thermal infrared, the chief problem is the limited sensitivity to low disk surface brightnesses when observing through atmospheric sky backgrounds. Improvements here will require large telescopes in space, and nulling interferometers on the ground. Millimeter and submillimeter observations have shown the most consistent ability to resolve dust disks, with a sensitivity down to 150 times our solar system's optical depth demonstrated in the SCUBA image of Vega (19). However, the spatial resolution now available at these wavelengths is many times worse than that achieved at the shorter wavelengths. Larger millimeter arrays with southern hemisphere sky coverage are needed.

Fortunately there are upcoming astronomical projects and facilities that will offer significant benefits to disk imaging science. One of these is NASA's Space Infrared Telescope Facility (SIRTF), scheduled for a 2002 launch. Even with its modest $0.85 \mathrm{~m}$ aperture, SIRTF will be able to detect and resolve dozens of nearby debris disks at $70 \mu \mathrm{m}$, down to optical depths less than 100 times the solar system's zodiacal light. Furthermore, SIRTF should photometrically detect thousands of new disks suitable for follow-up imaging studies. Particularly interesting will be nearby late-type stars possessing debris disks as optically thick as that of $\beta$ Pic, but which went undetected by IRAS due to the central star's low luminosity.

An ideal instrument to image the new disks uncovered by SIRTF will be the Atacama Large Millimeter Array (ALMA), which is expected to begin operation late in this decade. With 64 antennas of $12 \mathrm{~m}$ aperture spread across baselines extending to $10 \mathrm{~km}$, ALMA will achieve a spatial resolution of $0.03^{\prime \prime}(0.5 \mathrm{AU}$ at $\beta \mathrm{Pic} ; 4 \mathrm{AU}$ in nearby star-forming regions). ALMA will provide detailed maps of the density, kinematic, and chemical structure of protoplanetary disks in molecular line emission; and of the dust continuum emission of nearby debris disks. With no stellar contrast problem at these wavelengths, ALMA should map more disks in more detail than any prior astronomical facility.

In the optical and near-infrared, it does not appear that the Next Generation Space Telescope (NGST) or new AO systems on very large telescopes will offer major image contrast improvements. Current adaptive optics systems correct the wavefront to only $\lambda / 15$ or so, while HST has fixed wavefront errors of $\sim \lambda / 40$. These errors lead to unwanted scattering of light into a halo around the central star. Increases in aperture size, or in the number of actuators used to control the wavefront, yield only small improvements in image contrast. The key (and often unappreciated) requirement is precise control of the optical wavefront: errors must be reduced toward $\sim \lambda / 1000$ at the critical mid-spatial frequencies to enable direct imaging of exozodiacal dust at levels comparable to that of our own solar system. This goal will soon be achievable using a dedicated space telescope intrumented with a precision deformable mirror and a coronagraph. Absent this, only incremental progress can be expected in optical and near-infrared disk imaging in the coming decade. 


\section{References}

(1) Augereau, A., Lagrange, A., Mouillet, D., \& Ménard, F. 2001, A\&A, 365, 78

(2) Backman, D. \& Paresce, F. 1993, in Protostars \& Planets III, E. Levy and J. Lunine eds., Univ. of Arizona Press, Tuscon, pp. 1253-1304

(3) Bally, J., O’Dell, C.R., \& McCaughrean, M.J. 2000, AJ, 119, 2919

(4) Burrows, C.J. et al. 1996, ApJ, 473, 437

(5) Burrows, C.J. et al. 1995, BAAS, 187, 3205

(6) Close, L.M. et al. 1998, ApJ, 499, 883

(7) Close, L.M. et al. 1997, ApJ, 478, 766

(8) Dent, W., Walker, H., Holland, W., \& Greaves, J. 2000, MNRAS, 314, 702

(9) Dutrey, A. et al. 1998, A\&A, 338, L63

(10) Dutrey, A. et al. 1996, A\&A, 309, 493

(11) Duvert, G. et al. 2000, A\&A, 355, 165

(12) Duvert, G. et al. 1998, A\&A, 332, 867

(13) Grady, C.A. et al. 2000, ApJ, 544, 895

(14) Greaves, J.S. et al. 1998, ApJ, 506, L133

(15) Guilloteau, S., Dutrey, A., \& Simon, M. 1998, A\&A, 348, 570

(16) Guilloteau, S., \& Dutrey, A. 1998, A\&A339, 467

(17) Hayashi, M., Ohashi, N., \& Miyama, S. 1993, ApJ, 418, L71

(18) Heap, S.R. et al. 2000, ApJ, 539, 435

(19) Holland, W.S. et al. 1998, Nature, 392, 788

(20) Jayawardhana, R. et al. 1998, ApJ, 503, L79

(21) Kalas, P., \& Jewitt, D. 1996, AJ, 111, 1347

(22) Kalas, P., \& Jewitt, D. 1995, AJ, 110, 794

(23) Koerner, D., Ressler, M., Werner, M., \& Backman, D. 1998, ApJ, 503, L83

(24) Koerner, D.K., \& Sargent, A.I. 1995, AJ, 109, 2138

(25) Krist, J., Stapelfeldt, K., Ménard, F., Padgett, D., \& Burrows, C. 2000, ApJ, 538, 793

(26) Lay, O.P., Carlstrom, J.E., \& Hills, R.E. 1997, ApJ, 489, 917

(27) Liou, J.-C., \& Zook, H. 1999, AJ, 118, 580

(28) Mannings, V., \& Sargent, A.I. 2000 ApJ, 529, 391

(29) Mannings, V., \& Sargent, A.I. 1997 ApJ, 490, 792

(30) Mannings, V., Koerner, D.W., \& Sargent, A.I. 1997, Nature, 388, 555

(31) Marsh, K. et al. 1995, ApJ, 451, 777

(32) McCaughrean, M., Stapelfeldt, K., \& Close, L. 2000, in Protostars 6 Planets IV, Mannings, Boss, and Russell eds., Univ. of Arizona Press, Tucson, pp. 485-507

(33) Monin, J.-L., \& Bouvier, J. 2000, A\&A, 356, L75

(34) Mouillet, D., Larwood, J., Papaloizou, J., \& Lagrange, A. 1997, MNRAS, 292, 896

(35) Padgett, D.L., Brandner, W., Stapelfeldt, K.R., Strom, S.E., Terebey, S., \& Koerner, D. 1999, AJ, 117, 1490

(36) Pantin, E., Lagage, P.O., \& Artymowicz, P. 1997, A\&A, 327, 1123

(37) Roddier, C., Roddier, F., Northcott, M., Graves, J., \& Jim, K. 1996, ApJ, 463, 326

(38) Rodriguez, L.F. et al. 1998, Nature, 395, 355

(39) Schneider, G. et al. 2000, this volume

(40) Silber, J., Gledhill, T., Duchêne, G., \& Ménard, F. 2000, ApJ, 536, L89

(41) Simon, M., Dutrey, A., \& Guilloteau, S. 2001, ApJ, 545, 1034

(42) Smith, B., Fountain, J., \& Terrile, R. 1992, A\&A, 261, 499 
(43) Smith, B., \& Terrile, R. 1984, Science, 226, 1421

(44) Stapelfeldt, K. et al. 2001, in preparation

(45) Stapelfeldt, K. et al. 1999, ApJ, 516, L95

(46) Stapelfeldt, K., Krist, J., Ménard, F., Bouvier, J., Padgett, D., \& Burrows, C. 1998, ApJ, 502, L65

(47) Trilling, D., Brown, R., \& Rivkin, A. 2000, ApJ, 529, 499

(48) Wilner, D.J. et al. 2000, ApJ, 534, L101

(49) Wood, K., Crosas, M., \& Ghez, A. 1999, ApJ, 516, 335

(50) Wood, K., Kenyon, S., Whitney, B., \& Turnbull, M. 1998, ApJ, 497, 404 